\title{
Tandem overexpression of five human factors renders murine hepatocytes susceptible to hepatitis $C$ virus
}

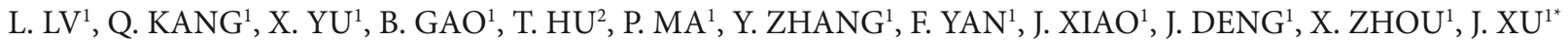 \\ ${ }^{1}$ Beijing Institute of Transfusion Medicine, Beijing 100850, P. R. China; ${ }^{2}$ Southwestern Medical School, UT Southwestern Medical \\ Center, Dallas, TX 75390, USA
}

Received August 11, 2014; accepted February 2, 2015

\begin{abstract}
Summary. - Development of mouse model of hepatitis C virus (HCV) infection has great significance in drug screening and vaccine research. The barriers of interspecies transmission of HCV are increasingly better understood. Human factors, namely low-density lipoprotein receptor (hLDLR), CD81 (hCD81), scavenger receptor class B type I (hSCARB1), occludin (hOCLN) and claudin 1 (hCLDN1) are all required for rendering mouse hepatocytes permissive to HCV. With the aim to humanize mouse hepatocytes we constructed two recombinant vectors tandemly expressing the first three and the last two HCV entry factors mentioned above, respectively. Cotransfection of mouse hepatocytes with these vectors made them permissive to HCV binding and entry. Tandem overexpression of hLDLR, hSCARB1, hCD81, hCLDN1 and hOCLN is a novel approach to tailoring mouse hepatocytes to HCV binding and entry which can be further used to establish a mouse model of HCV infection as a basis for developing antiviral drugs and vaccines.
\end{abstract}

Keywords: hepatitis C virus; mouse hepatocytes; human factors

\section{Introduction}

Hepatitis C virus (HCV), which is responsible for 170 million chronically infected patients, remains to be a worldwide threat (Safioleas and Manti, 2007). About 70\% or more patients develop liver cirrhosis or carcinoma which comprises the main causes of liver transplantation (Hsu et al., 2013; Mensa et al., 2013). Even though directly acting antivirals (DAA) in combination with pegIFN and ribavirin (RBV) leads to about $80 \%$ of cured (Rose et al., 2014), treatments targeting the early steps of HCV binding or entry processes are only supplemental treatments, especially for liver transplantation

*Corresponding author. E-mail: xujb201413@sina.com; phone: +86-10-66931964.

Abbreviations: $\mathrm{HCV}=$ hepatitis $\mathrm{C}$ virus; $\mathrm{hLDLR}=$ human low-density lipoprotein receptor; hCD81 = human CD81; hSCARB1 = human scavenger receptor class B type type I; hCLDN1 = human claudin $1 ;$ hOCLN = human occludin; $\mathrm{HCVcc}=$ cell culture-derived $\mathrm{HCV}$; HCVsd = serum-derived HCV and difficult-to-treat-patients. Binding or entry inhibitors are needed to restrain the prevalence of $\mathrm{HCV}$ infection.

However, effort to discover HCV binding or entry inhibitors has been hampered by the lack of animal model, which is of great use for HCV study or in development of HCV vaccine. HCV has strictly confined hosts, such as, humans and chimpanzees (Kolykhalov et al., 1997). Although chimpanzee is an excellent animal model for the development of effective drugs and vaccines against $\mathrm{HCV}$, ethical issues, limited availability, and high cost severely limit its use as a laboratory animal. Human hepatocyte chimeric immunodeficient mice have been developed by xenotransplantation, but they are vulnerable and the environmental and logistic needs are demanding (Ilan et al., 2002; Mercer et al., 2001). An alternative strategy is to humanize mouse tissues. In this regard, how to render mouse hepatocytes susceptible to HCV is the first obstacle that remains to be overcome.

Mouse hepatocyte cells are resistant to HCV entry, but do support virion replication, assembly and release (McCaffrey et al., 2002). Numerous factors exist on human hepatocyte 
surface, which determine the tropism of HCV. Of these factors, several key determinants required for HCV entry have been identified, including low-density lipoprotein receptor (LDLR) (Agnello et al., 1999), scavenger receptor class B type I (SCARB1) (Scarselli, 2002), tetraspanin (CD81) (Pileri et al., 1998), tight junction proteins claudin 1 (CLDN1) (Evans et al., 2007), and occludin (OCLN) (Ploss et al., 2009). HCV entry into the hepatocytes is a multistep, dynamic process which involves a temporal and spatial interaction of virion with entry factors. Adenoviral or transgenic expression of human entry factors permits HCV uptake in mouse hepatocytes. In this study, we attempted to improve humanized mouse hepatocytes permissible to serum-derived $\mathrm{HCV}$ (HCVsd) infection. Five putative HCV entry factors of human origin, namely, hLDLR, hSCARB1, hCD81, hCLDN1, and hOCLN, were selected and partitioned into two groups. One group included hLDLR-hSCARB1-hCD81 and the other hCLDN1-hOCLN. To facilitate their interaction with HCV virion and increase expression level, each group of entry factors was expressed in tandem in mouse hepatocytes. Mouse hepatocytes stably transduced with these HCV entry factors showed increased susceptibility to HCVsd binding and endocytosis. Our study provided evidence that these five human factors are required for HCVsd entry, and offered a promising strategy to construct HCVsd permissive mouse model.

\section{Materials and Methods}

Cells. 293FT cells (Invitrogen, USA) and mouse hepatoma cell line Hepa 1-6 (a generous gift from Shoujun Yuan, Beijing Institute of Radiation Medicine) were cultured in DMEM containing 10\% fetal bovine serum (FBS).

HCV-positive human serum. HCV-positive sera were obtained from Beijing 302 hospital under an institutional review boards (IRB) approved protocols. The pooled serum samples contained $10^{7}$ copies/ml of HCV RNA plus-strand as determined by Light Cycler TM (Roche).

Vector constructs. We have constructed pCDH-hLDLRhSCARB1-hCD81-GFP recombinant expression vector which expresses a fusion protein hLDLR-hSCARB1-hCD81 (Fig. 1). TGGGG GGGGS GGGGS linker was inserted between hLDLR and hSCARB1 and linker ASGGG GGGGS GGGGS between hSCARB1 and hCD81. hLDLR ORF was amplified from pcDNA3-hLDLR vector (Lv et al., 2004) with primers 5'CTA GTC TAG AGC CAC CAT GGG GCC CTG GGG CTG G and 5'CAT GAC CGG TCG CCA CGT CAT CCT CCA GAC TGA C. hSCARB1 ORF was amplified from human hepatoma HepG2 cells with primers 5'CTA GTC TAG AAC CGG TGG CGG TGG CGG TGG CGG AGG ATC CGG CGG AGG CGG CTC CGG CTG CTC CGC CAA AGC GCG and CTA GCT AGC CAG TTT TGC TTC CTG CAG CA. hCD81 ORF was amplified from Huh7.5.1 cells with primers 5'CTA GTC TAG AGC TAG CGG CGG GGG CGG TGG CGG AGG CTC CGG AGG AGG GGG ATC CGG AGT GGA GGG CTG CAC CAA and 5'CCG GAA TTC AGG GCC GGG ATT CTC CTC CAC GTC ACC GCA TGT TAG AAG ACT TCC TCT GCC CTC GTA CAC GGA GCT GTT CCG GAT GC. The three fragments were digested and inserted into pCDH-EF1-MCS-GFP vector to yield pCDH-hLDLRhSCARB1-hCD81-GFP recombinant vector.

Recombinant expression vector pCDH-hCLDN1-hOCLNDsRed expresses a fusion protein of hCLDN1-hOCLN. GGGGS GGGGS GGGAS linker was inserted between hCLDN1 and hOCLN to maintain individual formation. hCLDN1 and hOCLN were both (a)

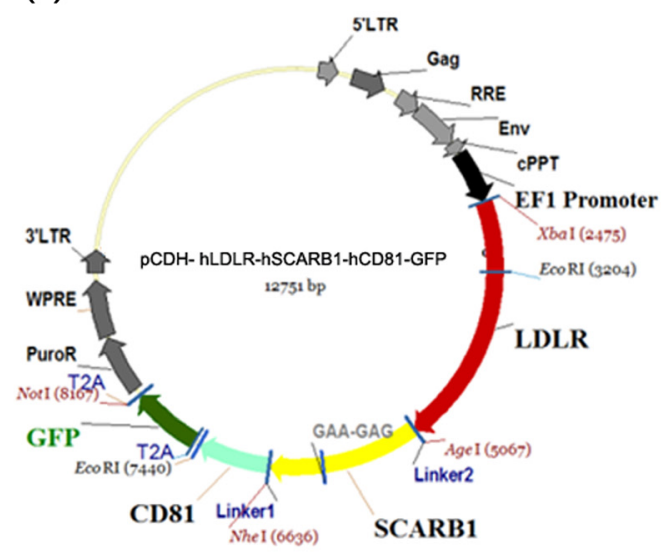

(b)

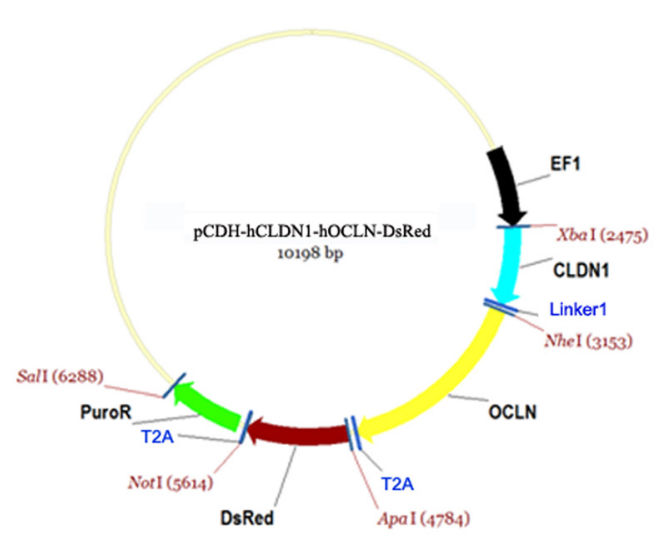

(c)

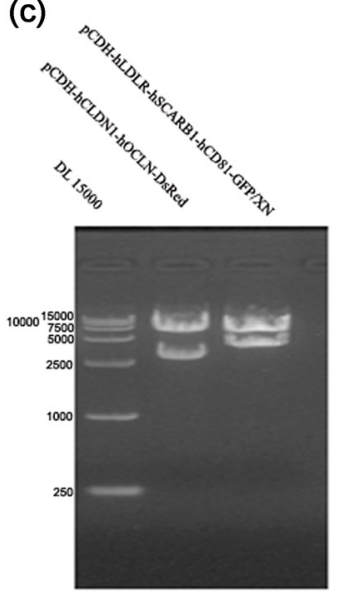

Fig. 1

Construction of recombinant expression vectors

(a) Map of vector expressing hLDLR-hSCARB1-hCD81 fusion protein. (b) Map of vector expressing hCLDN1-hOCLN fusion protein. (c) Restriction maps of vectors pCDH-hLDLR-hSCARB1-hCD81-GFP and pCDH-hCLDN1-hOCLN-DsRed. 
amplified from human hepatocytes QSG7701. hCLDN1 ORF was amplified with primers 5'GCT CTA GAA TGG CCA ACG CGG GGC T and 5'CTA GCT AGC GCC ACC GCC GGA TCC TCC GCC ACC GGA GCC GCC TCC GCC CAC GTA GTC TTT CCC. hOCLN ORF was amplified with primers 5' CTA GCT AGC ATG TCA TCC AGG CCT CTT and 5'AAT GGG CCC GAG GGC CGG GAT TCT CCT CCA CGT CAC CGC ATG TTA GAA GAC TTC CTC TGC CCT CTG TTT TCT GTC TAT C. The two fragments were digested and inserted into pCDH-MCS-T2A-DsRed vector to yield pCDH-hCLDN1-hOCLN-DsRed recombinant vector.

Mouse hepatocytes expressing HCV entry factors. Recombinant lentivirus expressing hLDLR-hSCARB1-hCD81-GFP and hCLDN1-hOCLN-DsRed were generated by cotransfection of 293 FT cells with vector RSV-REV, VSVG, and pMDLg/pRRE. Mouse hepatocytes were seeded at $1.5 \times 10^{5}$ cells per well in a six-well plate and infected for $24 \mathrm{hr}$ with the mixed-package lentivirus. The transduced cells were propagated in complete medium and transgenic mouse hepatocytes were sorted via flow cytometry (MoFlo cell sorter, DAKO, USA).

Multicolour flow cytometry. In order to sort transgenic cells containing hLDLR-hSCARB1-hCD81 (GFP) and hCLDN1-hOCLN (DsRed) we used multi-color flow cytometry.

Western blot analysis. Transgenic mouse hepatocytes were collected and lysed in RIPA buffer containing inhibitors of proteases for $30 \mathrm{~min}$ on ice. After sonication, samples were centrifuged and the protein concentration was determined using BCA protein assay (KangWei, China) according to the manufacturer's instruction. Normalized protein quantities were separated by $10 \%$ SDS-PAGE and blotted to PVDF membranes for incubation with hCLDN1 or hSCARB1-specific antibodies in western blot analysis. Detection of the chemiluminescent signal was performed with the super ECL western blotting detection kit (Applygen Technologies Inc., China).

HCVcc generation. Vector FL-J6/JFH-5'C19Rluc2AUbi (provided by C. Rice, Rockefeller University, NY) encoding full-length JFH-1 was used to generate RNA as previously described (Lindenbach et al., 2005). Briefly, RNA was in vitro transcribed from fulllength genomes using the MEGAscript T7 kit (Ambion, USA) and transduced into Huh-7.5 cells DMRIE-C (Invitrogen). Seventy-two hours post-transduction, supernatants were collected and stored immediately at $-80^{\circ} \mathrm{C}$.

HCV binding and entry assay. Cells were incubated with HCVsd or HCVcc for $1 \mathrm{hr}$ at $4^{\circ} \mathrm{C}$. Cells were either harvested to assess binding or moved to $37^{\circ} \mathrm{C}$ for $5 \mathrm{hr}$, followed by proteinase $\mathrm{K}$ cleavage of external virus to assess entry. Luciferase activity and HCV copy number was determined accordingly.

HCV RNA copies assay. HCV RNA copies were determined by Light Cycler TM (Roche, Switzerland) using real-time RT-PCR (Kehua, China).

Statistical analysis. Results were expressed as means \pm SD of at least three independent experiments. Statistical analyses were performed using the $\mathrm{x}^{2}$ test, and $\mathrm{P} \leq 0.05$ was considered statistically significant.

\section{Results}

\author{
Construction and characterization of recombinant ex- \\ pression vectors
}

Several studies have shown that hCD81 and hOCLN comprise the minimal human factors required for $\mathrm{HCV}$ entry in vitro (Ploss et al., 2009). However, transgenic mice expressing hCD81, hOCLN, hSCARB1 and hCLDN1 supported a more complete HCV life cycle (Dorner et al., 2011; 2013). Furthermore, hLDLR has been shown to play an important role in HCVsd infection of target cells in vivo (Liu et al., 2012; Lv et al., 2009). Taken together, in order to equip the mouse hepatocytes with the full suite of factors for HCV entry, we incorporated the full list of five human factors into mouse hepatocytes. Since HCV interacts with these factors through searching-and-binding, a tandem expression strategy was adopted to increase the vicinity of these factors on the membrane which maximally favors the entry of HCV. Considering that hLDLR, hSCARB1 and hCD81 present on the plasma membrane are associated with the early step of $\mathrm{HCV}$ binding whereas hOCLN and hCLDN1 are tight junction proteins involved in the later steps, two groups of factors were respectively inserted into two plasmids. Linkers were inserted between two adjacent proteins to preserve the original protein conformation and reduce the protein-protein interference. Fluorescent selective markers, GFP and DsRed, were tagged to the C-terminal of these two recombinant proteins respectively, thereby, constructing two recombinant expression vectors, pCDH-hLDLR-hSCARB1-hCD81-GFP (Fig. 1a) and pCDH-hCLDN1-hOCLN-DsRed (Fig. 1b), using molecular cloning. The insertion of target genes into vector was verified by restriction enzyme digestion (Fig. 1c).

\section{Establishment of transgenic mouse hepatocytes}

Then we attempted to establish hepatoma cell line which stably expresses these five human factors for HCV entry. Two recombinant expression vectors were generated and then transfected into Hepa 1-6 cells. The cells that stably express these factors were sorted through flow cytometry based on the fluorescent marker. About 95\% of transgenic mouse hepatocytes expressed GFP, which also indicated the expression of the three proteins encoded by the inserted genes in vector pCDH-hLDLR-hSCARB1-hCD81-GFP; about 49.3\% hepatocytes expressed DsRed, suggesting the expression of two tight junction proteins encoded by pCDH-hCLDN1hOCLN-DsRed; and 97.2\% cells expressed all five human factors carried by both recombinant lentiviruses (Fig. 2a). Confocal microscopy revealed that the recombinant proteins were expressed predominantly on the plasma membrane as well as in the cytoplasm (Fig. 2b,c). Western blot further veri- 
(a)
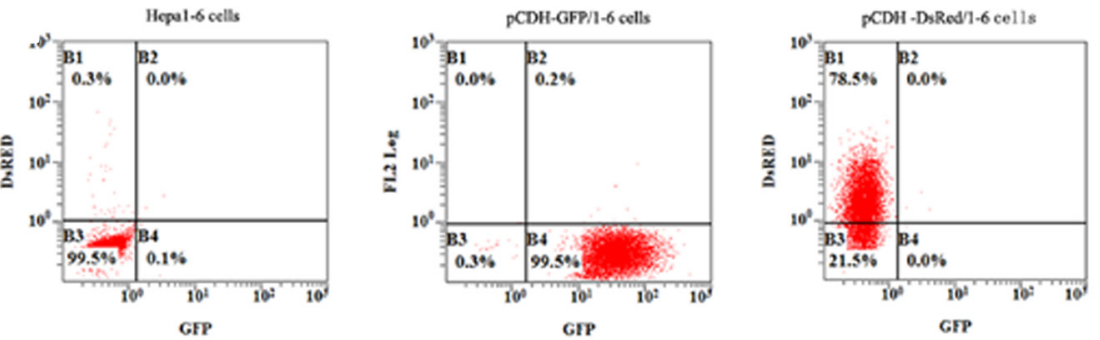

GRP
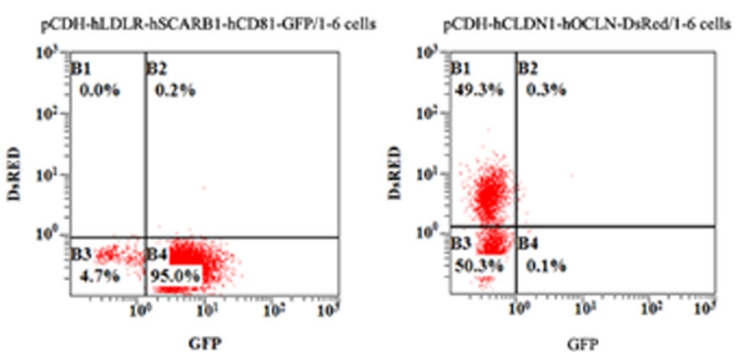

PCDH-LLDLR-HSCARB1-HCDS1-GFP* DCDH-BCLDNI-SOCLN-DiRed/1-6 cells

(b)
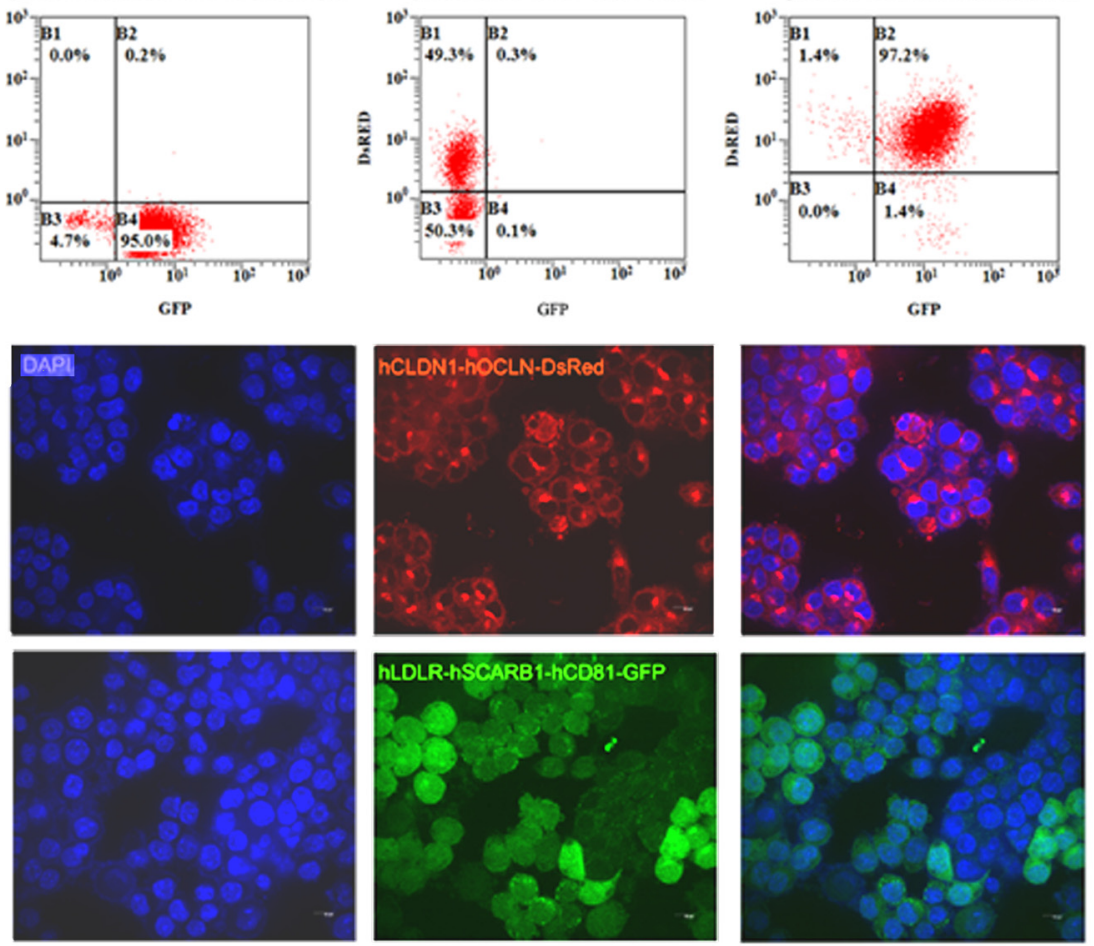

(c)

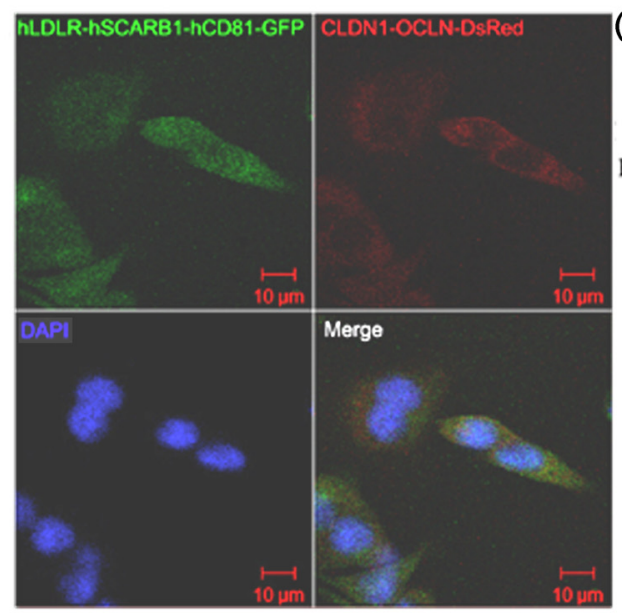

\section{(d)}
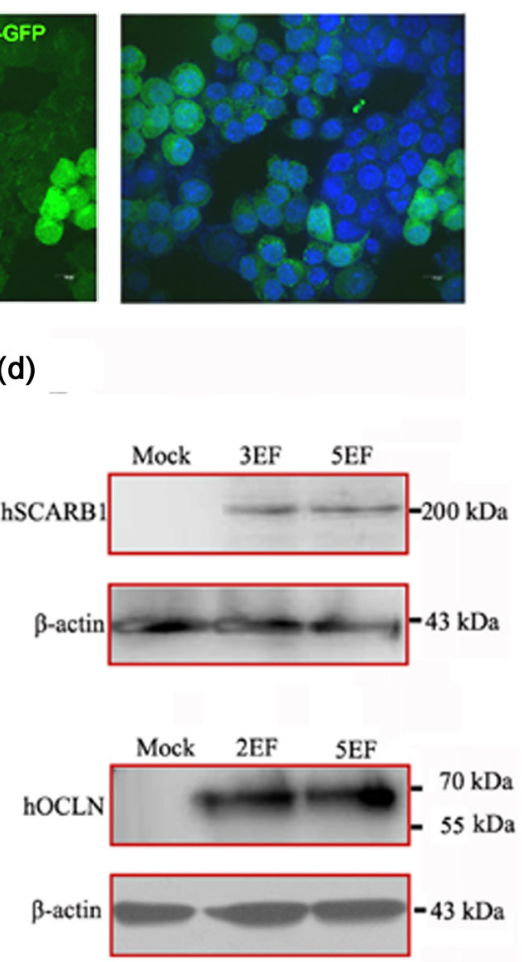

Fig. 2

Expression of human HCV entry factors in transgenic cells

(a) Flow cytometry of transgenic cells. (b) Confocal microscopy of cells transfected with recombinant expression vectors pCDH-hCLDN1-hOCLN-DsRed/ upper row and pCDH-hLDLR-hSCARB1-hCD81-GFP/lower row. (c) Confocal microscopy of cells stably expressing hLDLR-hSCARB1-hCD81 in green, and hCLDN1- hOCLN in red. (d) Western blot analysis of expression of hSCARB1 and hOCLN. 
fied the expression of five HCV factors, two fusion proteins hLDLR-hSCARB1-hCD81 and hCLDN1-hOCLN in transgenic mouse hepatocytes (Fig. 2d). The transgenic cells were able to express the desired factors on their surface during several passages in the presence of selection pressure (data not shown). Thus, transgenic mouse hepatocytes harboring the full list of key HCV factors were established, and these proteins were brought closer spatially to create a favorable environment for HCV entry.

\section{Overexpression of HCV entry factors correlates with enhanced $\mathrm{HCV}$-cell binding}

The early viral attachment is the first step during the virus infection, and during this process HCV-factor binding is responsible for initiating a series of events that lead to subsequent viral entry. To determine the effect of tandem expression of human factors on HCV binding, we examined the HCV binding ability in the transgenic mouse hepatocytes. Overexpression of three plasma membrane localized factors, hLDLR, hSCARB1, and hCD81, significantly enhanced the binding ability of HCVsd particles to mouse hepatocytes, compared to non-transduced cells. However, neither overexpression of tight junction proteins alone nor in combination with expression of other three human factors had obvious effect on HCV binding ability (Fig. 3a). The data showed the important role of hLDLR in the process of HCVsd binding. $\mathrm{HCVcc}$ particle binding was not altered by the expression of either group of human factors alone, or together (Fig. 3b). However, caution should be taken when interpreting the results since unspecific binding may occur. Nevertheless, these observations suggest that the tandem expression of at least three of these five human factors, if not all, supports HCV binding.

\section{Increased HCV endocytosis in transgenic cells}

As mentioned above, an unspecific binding of virus to the cell surface may occur. We have reported that in mouse hepatocytes, this binding involves unknown factors on the cell membrane, and is not necessarily followed by virus entry (Lv et al., 2009). In comparison with the binding process, viral internalization is more factor-dependent and host-specific, which strictly relies on the presence of several human factors. We thus performed HCV entry assay, which ruled out the possibility of unspecific binding. Transduced cells were infected with $\mathrm{HCV}$ sd at $4^{\circ} \mathrm{C}$ for $1 \mathrm{hr}$ followed by $37^{\circ} \mathrm{C}$ for $5 \mathrm{hr}$. In order to eradicate the non-specific binding virus, the infected cells were then treated with proteinase $\mathrm{K}$. The cells were harvested after $72 \mathrm{hr}$ for RNA copies assay. Transduced cells, whether they expressed one or both groups of the human factors, showed a significant increase in HCVsd internalization compared to the parental cells. The
HCVsd binding ability to the transgenic mouse hepatocytes was comparable to that of Huh 7.5.1 (about $10^{6}$ RNA copies), which suggests that the modified mouse hepatocytes support HCV entry to the same extent as human hepatocytes. Consistently, transgenic cells also showed enhanced HCVcc internalization (Fig. 4). In both cases of HCVsd and HCVcc infection, the introduction of all five human factors rendered highest permissibility to HCV internalization, followed by the expression of three plasma membrane resident factors, and then by two tight junction proteins. These data demonstrate that tandem expression of human factors increases HCV internalization into mouse hepatocytes, and that humanized mouse hepatocytes allow HCV entry.

\section{Discussion}

We previously reported that human factors on cell surface are required for endocytosis of HCV (Lv et al., 2009). Ploss, et al. introduced hCD81 and hOCLN into mouse hepatocytes and demonstrated that they are the minimal human factors required for HCV entry in vitro (Ploss et al., 2009). Dorner et al. humanized mouse liver by introducing four human factors, but the efficiency of expressing all four factors was low (Dorner et al., 2011). In this study, we took a novel approach to express five human key factors for HCV entry in mouse hepatocytes. These factors were divided into two groups based on their localization and expressed in tandem. This approach ensures the efficiency of simultaneous expression of five transfected proteins, and most importantly, maximally facilitates viral interaction because of spatial vicinity. Mouse hepatocytes expressing three plasma membrane resident human factors increased HCVsd binding, while cells expressing other human factors or all five showed no difference in HCV binding, probably due to unspecific binding compared to the control. However, the HCV binding ability to all the transduced cells was similar to that of the HCV permissive human cell line Huh7.5.1, which suggests that they support HCV binding. After virion binding, HCV entry can only happen when it encounters specific entry factors, and internalization is not initiated until full engagement of sufficient factors. The virus-factor complex is then transported to tight junctions, constituting the final step for viral internalization into the cell. Thus, we hypothesize that viral internalization depends on several specific human factors and is a more reliable indicator for assessing HCV entry. In agreement with our hypothesis, the tandem expression of human factors greatly boosted HCV internalization in mouse hepatocytes. Most intriguingly, the HCV RNA levels were expressed for up to $10^{6}$ copies in five human factors transduced mouse hepatocytes, suggesting that the susceptibility of our transgenic mouse hepatocytes are comparable to that of the canonical HCV permissive Huh7.5.1 cells. The increase in binding and 
Fig. 3

Effects of tandemly overexpressed human HCV entry factors on HCVsd and $\mathrm{HCVcc}$ binding to transgenic cells Real-time PCR. Binding of HCVsd (a) and HCVcc (b) to transgenic cells.

\section{Fig. 4}

Effects of tandemly overexpressed human HCV entry factors on entry of HCVsd and HCVcc into transgenic cells (a) Transgenic cells were incubated with HCVsd and virus entry was determined by real-time PCR. (b) Transgenic cells were incubated with $\mathrm{HCVcc}$ and virus entry was determined by luciferase assay. (a) LDLR SCARBI CD81 OCLN CLDN

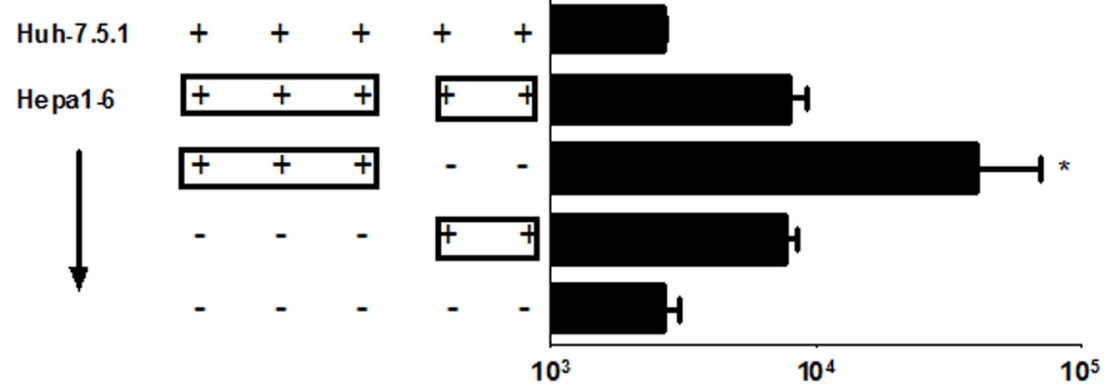

Serum-derived HCV binding as say (RNA copies)

(b) LDLR SCARBI CD81 OCLN CLDN

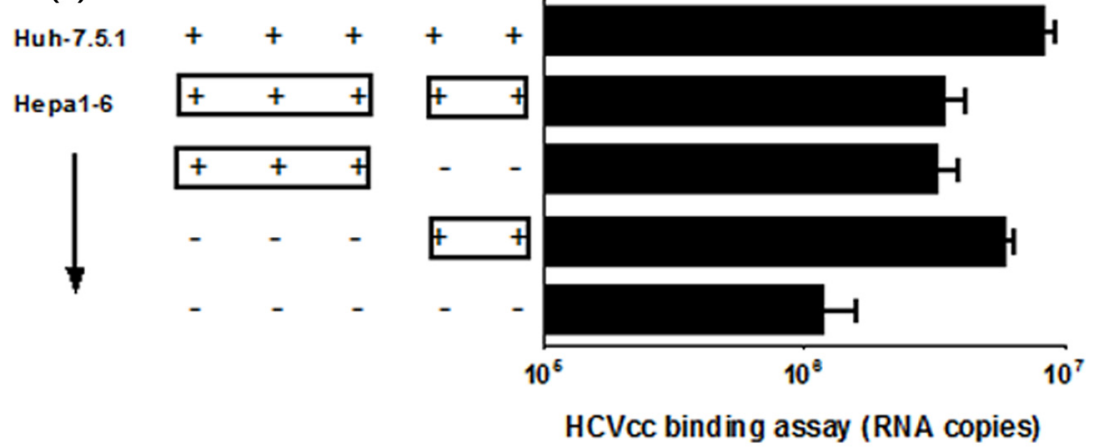

(a) LDLR SCARBI CD81 OCLN CLNN

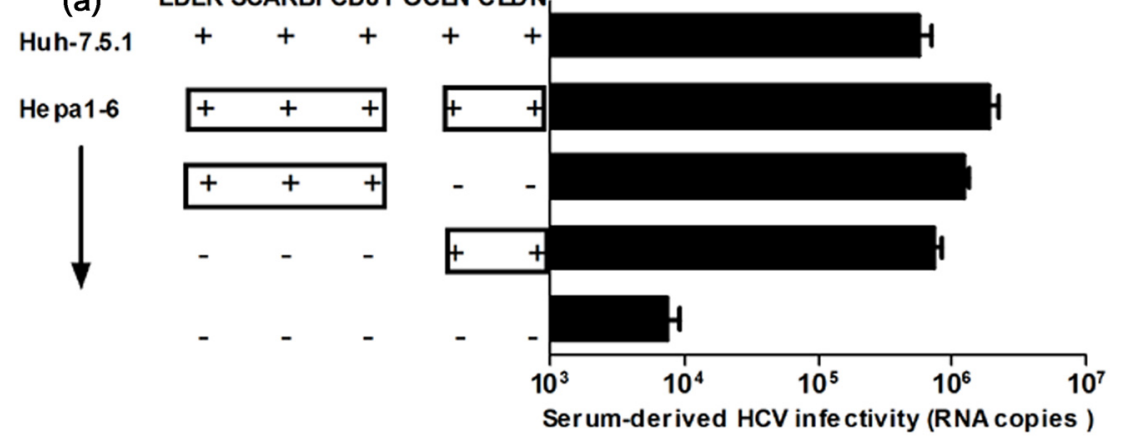

(b) LDLR SCARBI CD81 OCLN CLNN

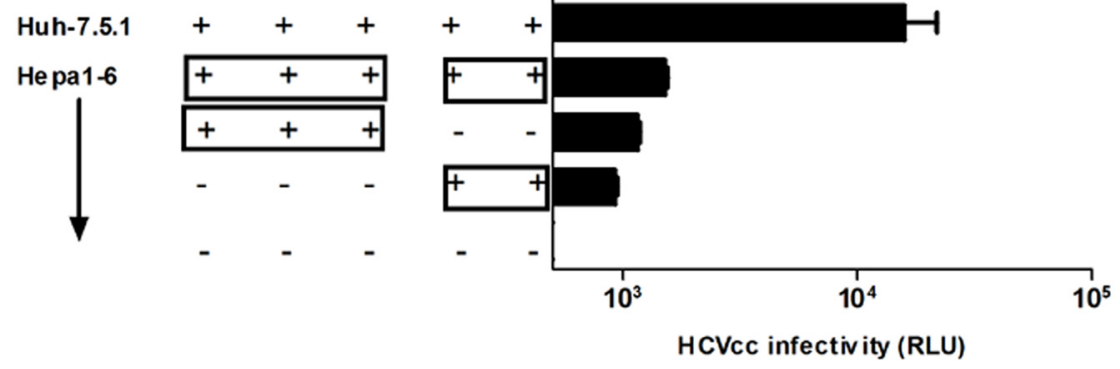


internalization was abolished by the adding of corresponding antibodies against human factors, which confirms that it is the human factors that mediate viral binding and subsequent internalization. Antibodies blocked the HCV binding as well as internalization process (data not shown). Taken together, these data suggest that the presence of human factors on the surface of mouse hepatoma Hepa 1-6 cells resulted in the sensitivity to HCV infection.

In this study, we established humanized mouse hepatocytes that posses the full suite of required factors for HCV especially HCVsd entry. These mouse hepatocytes show markedly elevated permissiveness to HCVsd entry. Although the distribution of these human factors needs to be confirmed, this tandem expression approach offers a potential for tailoring mouse hepatocytes to HCV entry, which can be further used to transplant into mouse livers. This is a significant step towards constructing HCVsd mouse model, which is the basis for developing antiviral drugs and vaccines.

Acknowledgements. This work was supported by the grant No. 81101296 from the National Natural Science Foundation of China. The authors thank Prof. Charles M. Rice from Rockefeller University for his generous gift of vector FL-J6/JFH-5'C19Rluc2AUbi.

\section{References}

Agnello V, Abel G, Elfahal M, Knight GB, Zhang QX (1999): Hepatitis $C$ virus and other flaviviridae viruses enter cells via low density lipoprotein receptor. Proc. Natl. Acad. Sci. USA 96, 12766-12771. http://dx.doi.org/10.1073/pnas.96.22.12766

Dorner M, Horwitz JA, Donovan BM, Labitt RN, Budell WC, Friling T, Vogt A, CataneseMT, Satoh T, Kawai T, Akira S, Law M, Rice CM, Ploss A (2013): Completion of the entire hepatitis $\mathrm{C}$ virus life cycle in genetically humanized mice. Nature 501, 237-241. http://dx.doi.org/10.1038/nature12427

Dorner M, Horwitz JA, Robbins JB, Barry, WT, Feng Q, Mu K, Jones CT, Schoggins JW, Catanese MT, Burton DR, Law M, Rice CM, Ploss A (2011): A genetically humanized mouse model for hepatitis $C$ virus infection. Nature 474, 208-211. http://dx.doi.org/10.1038/nature10168

Evans MJ, von Hahn T, Tscherne DM, Syder AJ, Panis M, Wolk B, Hatziioannou T. McKeating JA, Bieniasz PD, Rice CM (2007): Claudin-1 is a hepatitis $C$ virus co-receptor required for a late step in entry. Nature 446, 801-805. http://dx.doi.org/10.1038/nature05654

Hsu SH, Yeh ML, Wang SN (2013): New insights in recurrent HCV infection after liver transplantation. Clin. Dev. Immunol.2013, 890517. http://dx.doi. org $/ 10.1155 / 2013 / 890517$

Ilan E, Arazi J, Nussbaum O, Zauberman A, Eren R, Lubin I, Neville L, Ben-Moshe O, Kischitzky A, Litchi A, Margalit I, Gopher J, Mounir S, Cai W, Daudi N, Eid A, Jurim O, Czerniak A, Galun E, Dagan S (2002): The hepatitis C virus (HCV)-Trimera mouse: a model for evaluation of agents against HCV. J. Infect. Dis. 185, 153-161. http:// dx.doi.org/10.1086/338266

Kolykhalov AA, Agapov EV, Blight KJ, Mihalik K, Feinstone SM, Rice CM (1997): Transmission of hepatitis C by intrahepatic inoculation with transcribed RNA. Science 277, 570-574. http://dx.doi.org/10.1126/science.277.5325.570

Lindenbach BD, Evans MJ, Syder AJ, WolkB, Tellinghuisen TL, Liu CC, MaruyamaT, Hynes RO, Burton DR, McKeating JA, Rice CM (2005): Complete replication of hepatitis C virus in cell culture. Science 309, 623-626. http://dx.doi. org/10.1126/science. 1114016

Liu S, McCormick KD, Zhao W, Zhao T, Fan D, Wang T (2012): Human apolipoprotein E peptides inhibit hepatitis $C$ virus entry by blocking virus binding. Hepatology $56,484-491$. http://dx.doi.org/10.1002/hep. 25665

Lv LP, Jia SZ, Wang QL, Zhang YY, Zhou H, Xu JB (2009): Hepatitis $C$ virus infection of mouse hepatoma cell expressing human CD81 or LDLR. Acta Virol. 53, 185-189. http:// dx.doi.org/10.4149/av $2009 \quad 03 \quad 185$

Lv LP, Jia SZ, Wang HP, Zhan LS, Wang QL (2004): Cloning of human low density lipoprotein receptor gene and its expression in Hepa1-6 cells. Bull. Acad. Mil. Med. Sci. 28, 212-215.

McCaffrey AP, Ohashi K, Meuse L, Shen S, Lancaster AM, Lukavsky PJ, Sarnow P, Kay MA (2002): Determinants of hepatitis $\mathrm{C}$ translational initiation in vitro, in cultured cells and mice. Mol. Ther. 5, 676-684. http://dx.doi.org/10.1006/ mthe.2002.0600

Mensa L, Perez-del-Pulgar S, Crespo G, Koutsoudakis G, FernandezCarrillo C, Coto-Llerena M, Miquel R, Allende H, Castells L, Navasa M, Forns X (2013): Imaging of hepatitis C virus infection in liver grafts after liver transplantation. J. Hepatol. 59, 271-278. http://dx.doi.org/10.1016/j.jhep.2013.03.023

Mercer DF, Schiller DE, Elliott JF, Douglas DN, Hao C, Rinfret A, Addison WR, Fischer KP, Churchill TA, Lakey JR, Tyrrell DL, Kneteman NM (2001): Hepatitis C virus replication in mice with chimeric human livers. Nat. Med. 7, 927-933. http://dx.doi.org/10.1038/90968

Pileri P, Uematsu Y, Campagnoli S, Galli G, Falugi F, Petracca R, Weiner AJ, Houghton M, Rosa D, Grandi G, Abrignani S (1998): Binding of hepatitis C virus to CD81. Science 282, 938-941. http://dx.doi.org/10.1126/ science.282.5390.938

Ploss A, Evans MJ, Gaysinskaya VA, Panis M, You H, de Jong YP, Rice CM (2009): Human occludin is a hepatitis C virus entry factor required for infection of mouse cells. Nature 457, 882-886. http://dx.doi.org/10.1038/nature07684

Rose L, Bias TE, Mathias CB, Trooskin SB, Fong JJ (2014): Sofosbuvir: A nucleotide NS5B inhibitor for the treatment of chronic hepatitis Cinfection. Ann. Pharmacother. 48, 1019-1029. http://dx.doi.org/10.1177/1060028014534194

Safioleas MC, Manti CP (2007): Hepatitis C. An update. Saudi Med. J. 28, 1001-1006.

Scarselli E AH, Cerino R, Roccasecca RM, Acali S, Filocamo G, Traboni C, Nicosia A, Cortese R, Vitelli A (2002): The human scavenger receptor class $\mathrm{B}$ type $\mathrm{I}$ is a novel candidate receptor for the hepatitis C virus. EMBO J. 21, 5017-5025. http://dx.doi.org/10.1093/emboj/cdf529 